\title{
Ocular toxoplasmosis: the influence of patient age
}

\author{
Gary N Holland \\ Ocular Inflammatory Disease Center, Jules Stein Eye Institute, Department of Ophthalmology, David Geffen School of Medicine at UCLA, \\ University of California - Los Angeles, 100 Stein Plaza, 90095-7003 Los Angeles, CA, USA
}

The influence of patient age on various features of ocular toxoplasmosis has been a subject of study for many years. The age at which Toxoplasma gondii infection occurs in different populations is related to socioeconomic factors and studies suggest that ocular toxoplasmosis is a more severe disease at the extremes of age. The prevalence of ocular involvement is markedly different between individuals with congenital and those with post-natally acquired infections. Even among those with post-natally acquired infections, age influences the risk and timing of ocular involvement. The severity of toxoplasmic retinochoroiditis (in terms of lesion size, location and associated inflammation) is also affected by patient age at the time of initial infection or recurrence. The risk of recurrent toxoplasmic retinochoroiditis is influenced by age at the time of initial infection and age at most recent episode of active disease. Understanding of relationships between ocular toxoplasmosis and patient age is incomplete; evidence has often been indirect and in some cases conflicting. The influence of patient age on ocular toxoplasmosis should be studied in a systematic manner to provide a better understanding of disease mechanisms and to provide clinical information that can used to establish better strategies for disease treatment and prevention.

Key words: age - elderly - eye - recurrence - retinochoroiditis - toxoplasmosis

Age has always been a dominant theme in discussions about ocular toxoplasmosis, which is the most common infection of the retina in general populations worldwide. Ocular disease caused by Toxoplasma gondii was first described in 1923, in an infant with congenital infection (Jankû 1923), but it was not until a 1939 report by Wolf et al. that congenital toxoplasmosis with ocular involvement was widely accepted as a human disease. For more than a decade, toxoplasmic retinochoroiditis was thought to be a disease of newborns only. In the early 1950s, suspicion grew in many countries around the world, including Brazil, Germany (Rieger 1951) and Sweden (Wising 1952), that ocular toxoplasmosis could occur in adults as well. In 1952, a landmark paper by Wilder confirmed the presence of ocular toxoplasmosis in a series of adult patients with retinal lesions. Even so, it was assumed for many years that, among adults, ocular toxoplasmosis was a late sign of (usually asymptomatic) congenital infection. More recently, it has been argued convincingly that most cases of ocular toxoplasmosis are, in fact, attributable to post-natally acquired $T$. gondii infections (Holland 1999, 2003, 2004, Gilbert \& Stanford 2000).

As we enter a second century of research dealing with $T$. gondii, there is an appropriate emphasis on parasite genetics as a major determinant of ocular disease

Financial support: Research to Prevent Blindness, Inc (New York, NY), the Skirball Foundation (New York, NY) and the Vernon O. Underwood Family Endowed Professorship of Ophthalmology (David Geffen School of Medicine at UCLA, Los Angeles, CA).

+ Corresponding author: uveitis@jsei.ucla.edu

Received 10 October 2008

Accepted 3 February 2009 severity. Nevertheless, it is important to remember that host factors can also influence disease manifestations; one such factor is patient age. Risk of ocular involvement, as well as the severity of ocular disease when it does occur, varies between different age groups. This article summarizes information from a variety of sources to provide an overview of the relationship between ocular toxoplasmosis and patient age.

The majority of articles cited in this review were identified by searching PubMed for the years 1966-2008, using the following key words: toxoplasmosis, eye, age and elderly. Other articles were identified from the reference lists of articles found by the PubMed search.

\section{Prevalence of disease}

The most obvious influence of patient age on ocular toxoplasmosis is the marked difference in prevalence of ocular involvement between those with congenital infections and those who were infected post-natally. The risk of ocular disease among children with congenital T. gondii infections varies, depending on a number of factors, including the trimester of pregnancy when infection occurs and whether or not treatment was administered to the mother during pregnancy, but it has been reported to be as high as $85 \%$ (Couvreur \& Desmonts 1962, Stagno et al. 1977, Wilson et al. 1980), even among children who have subclinical infections at birth. The high risk of ocular involvement has been attributed to immaturity of the fetal immune system. In some patients with documented infections at birth, ocular lesions may not develop for years after birth (Koppe et al. 1986). Late ocular involvement has been attributed to reactivation of tissue cysts that have been present in normal-appearing retina since birth; in such cases, it is only with reactivation that there is a sufficient area of retinal necrosis to be identified clinically. 
In general, the prevalence of ocular involvement among people with post-natally acquired infections is much lower than with congenital infections. In the United States, ocular toxoplasmosis is believed to occur in approximately $2 \%$ of sero-positive individuals (Holland 2003). In other areas of the world, the prevalence is higher; in Southern Brazil, for example, where most T. gondii-infected individuals are believed to have been infected after birth, the reported prevalence of ocular involvement is approximately $18 \%$ (Silveira et al. 1988, Glasner et al. 1992). Similar prevalences of ocular involvement have been reported in several epidemics of $T$. gondii infection (Stagno et al. 1980, Burnett et al. 1998, de Moura et al. 2006). These higher prevalences have been attributed to infection with more virulent parasite genotypes. Individuals with post-natally acquired $T$. gondii infections also can first develop ocular lesions (in normal-appearing retina) years later, as with congenital infections (Bosch-Driessen \& Rothova 1999, Holland et al. 1999, Ongkosuwito et al. 1999, Silveira et al. 2001). Despite these facts, the cumulative prevalence of ocular toxoplasmosis associated with post-natally acquired infections never approaches the prevalence reported for congenital disease.

The prevalence of ocular toxoplasmosis in a community will therefore depend, in part, on the relative proportion of congenital and post-natally acquired infections. The proportion of congenital infections varies geographically, which has been attributed to variations in the age at which initial T. gondii infections occur in different populations and the proportion of women who remain sero-negative during their child-bearing years, and are therefore at risk for initial infection during pregnancy.

In the United States, the age-adjusted prevalence of individuals with anti-T. gondii $\operatorname{IgG}$ antibodies is $22.5 \%$, based on testing of serum collected in conjunction with the Third National Health and Nutrition Examination Survey (NHANES III), performed during the period 1988-1994 (Jones et al. 2001). In the United States, seroprevalence increases with age (Jones et al. 2001). The risk of infection in France appears to be constant across different age groups (Papoz et al. 1986) and as a result, seroprevalence in France also continues to increase with age (Carme \& Tirard-Fleury 1996), as in the United States (data also available from the Bulletin Epidemiologique Hebdomadaire thematique 14-15/8 April 2008, Institut de Veille Sanitaire). In contrast to the situation in the United States, $85 \%$ of the population in some areas of Southern Brazil is sero-positive and infections tend to be acquired in childhood (Glasner et al. 1992). Seroprevalence in Panama also rises more rapidly during the childhood years than in older age groups (Sousa et al. 1988), indicating decreased risk of infection in older individuals. These different patterns might reflect a greater proportion of infections in Panama from soil exposure, which is likely to be more common among children than among adults. By tradition, meat is cooked more thoroughly before eating in Panama than in France (Jones et al. 2001).

Seroprevalence for $T$. gondii infection has dropped among women of child-bearing age in the United States from $31.7 \%$ in the period 1967-1969 (Kimball et al. 1971) to $15 \%$ in $1988-1994$ (Jones et al. 2001). It is assumed that the same is true for the general population. There has been a substantially greater drop in the prevalence of congenital $T$. gondii infections during approximately the same interval, from one per 1,000 live births during the 1960's and the 1970's (Kimball et al. 1971, Alford et al. 1974) to 0.8 per 10,000 live births in the 1990's (Guerina et al. 1994). If one assumes that the frequency of ocular involvement has remained constant with both congenital and post-natally acquired $T$. gondii infections, then the proportion of patients with ocular disease attributable to post-natally acquired infections is rising. It is also possible that antimicrobial treatment of newborns with congenital T. gondii infection during the first year of life, as advocated by many authorities (McAuley et al. 1994, Brezin et al. 2003) may actually have lowered the frequency of ocular involvement in this population. Based on such facts, Gilbert and Stanford (2000) have argued that two-thirds of patients with ocular toxoplasmosis in the United Kingdom acquired their $T$. gondii infections post-natally, based on seroprevalence of $T$. gondii infection in the general population and rates of congenital disease. Similar arguments have been made for the United States, as well (Holland 1999, 2003, 2004).

The distribution of active toxoplasmic retinochoroiditis episodes in relation to patient age has also been a subject of study and has been remarkably similar in many reports (Friedmann \& Knox 1969, Gilbert et al. 1999, Bosch-Driessen et al. 2002). Patients in their second through fourth decades of life account for most observed episodes. In 1969, Friedmann and Knox found that the mean age of "initial episodes" of ocular toxoplasmosis in the Eastern United States was 25.3 years (median within the 20-24 year age group; mode 10-14 year age group). In 1999, Gilbert et al. found that the mean age for "first episodes" among patients in England was 26.5 years (mean age for all episodes was 31.1 years). Bosch-Driessen et al. (2002) found that 215 (78\%) of 274 observed episodes of active ocular toxoplasmosis in the Netherlands were in patients aged 15-45 years (mean age 31.1 years). Methodological differences make it difficult to compare these series directly, but the fact that the same basic pattern has been reported from different clinical settings during different time periods suggests that there is a true relationship between episodes of active disease and relatively young age.

A number of factors may contribute to the observed patterns. Friedmann and Knox (1969) hypothesized that hormonal changes that occur during adolescence account for a higher rate of recurrent disease. At the time of their report, it was assumed that most observed episodes were recurrences of congenital disease; however, it is now known that, depending on socioeconomic factors, post-natal $T$. gondii infection can occur throughout childhood (Stagno \& Thiermann 1973). If most cases of ocular toxoplasmosis are actually associated with postnatally acquired infection, the low number of patients with active toxoplasmic retinochoroiditis who are under 10 years of age in the United States and Europe may be attributable to the low prevalence of infection in that age group. Young children who do have active disease may fail to report symptoms, lowering the prevalence of ocular toxoplasmosis identified for that age group. 
In some areas of the world, including Southern Brazil, adolescents and young adults are known to have the highest rates of $T$. gondii infection (Silveira et al. 1988, 2001, Glasner et al. 1992), which might explain the larger number of observed episodes of ocular disease in those age groups. It is possible that new infections occur most frequently among younger patients in the United States as well. Although the cross-sectional NHANES III study showed a steady rise of seroprevalence with increasing age in the United States (Jones et al. 2001), sero-prevalence does not necessarily reflect the age at which infection occurs. If risk of infection is now lower for all age groups than in the past, a cohort effect could explain the reported relationship between seroprevalence and age; higher seroprevalence in older groups would reflect infection that occurred when that cohort was younger and the risk of infection was higher. A drop in the rate of recurrences over time, as discussed below, could then be one contributing factor to the lower number of observed episodes in older age groups. Interactions between age and other factors are complex and will require continued study to understand observed patterns.

\section{Age as a risk factor}

Even among those without congenital $T$. gondii infections, risk of infection and risk of ocular involvement appear to vary with age after birth. A 1976 epidemic that affected many members of a single family in the state of Alabama primarily involved the family's children, possibly related to age-specific activities, such as geophagia (Stagno et al. 1980).

The substantially lower number of observed episodes of active toxoplasmic retinochoroiditis among older age groups in the publications cited above (Friedmann \& Knox 1969, Gilbert et al. 1999, Bosch-Driessen et al. 2002) should not be interpreted to mean that the "risk" of ocular disease after recently acquired $T$. gondii infection decreases with age. An assessment of risk would also require knowledge of the age distribution of the total populations from which the cases were drawn and the seroprevalence for each age group, which was not provided in any of the publications. Data from other studies suggest that older patients actually have a higher risk of ocular involvement following recently acquired $T$. gondii infection than younger patients. Researchers in the Netherlands, for example, have found that most patients with ocular toxoplasmosis who have serologic evidence of recent infection are older (Ronday et al. 1995, Ongkosuwito et al. 1999, Bosch-Driessen et al. 2002). Patients whose ocular toxoplasmosis was first seen when they had serologic evidence of remotely acquired infection had a mean age of 29.9 years, while patients whose ocular toxoplasmosis was first seen while they had serologic evidence of recent infection had a mean age of 50.6 years (Bosch-Driessen et al. 2002). Also, patients with primary retinal lesions (defined as those not associated with pre-existing retinochoroidal scars) were older than those with recurrent lesions (Ongkosuwito et al. 1999, BoschDriessen et al. 2002). In a 1995 epidemic of T. gondii infection in Victoria, British Columbia, Canada, the mean age of infected patients without retinal lesions was ap- proximately 28 years, whereas the mean age of infected individuals with eye disease was 54 years (Burnett et al. 1998). Among 22 patients with ocular toxoplasmosis and serologic evidence of recent $T$. gondii infection in a study by Montoya and Remington (1996), the mean age was similar (50.2 years; median 51.5 years; range 16-79 years); cases were presumably associated with endemic disease in the United States. The consistency of these various observations strongly suggest that older patients have a higher risk of developing ocular lesions following recently acquired $T$. gondii infection, although this relationship cannot be confirmed definitively on the basis of these data alone.

Other evidence, from Brazil, supports an increased risk of ocular involvement among older patients following $T$. gondii infection. In contrast to the North American and European studies cited above (Friedmann \& Knox 1969, Gilbert et al. 1999, Bosch-Driessen et al. 2002), the prevalence of ocular disease in Southern Brazil is higher in successively older age groups (Glasner et al. 1992). Another, more recent study from Brazil showed that older age was the major risk factor for ocular disease among household members infected with $T$. gondii (Portela et al. 2004); the prevalence of ocular toxoplasmosis was significantly higher among those older than 50 years. Age has been among the epidemiologic risk factors for early development of ocular lesions among individuals with serologic evidence of recently-acquired $T$. gondii infection in Southern Brazil. In a retrospective study from the area of Erechim, Brazil, the risk of developing retinal lesions during the first two years after post-natally acquired T. gondii infection was greater among patients older than 40 years of age than among those who are younger $(p=$ 0.095, Kaplan-Meier analysis; Claudio Silveira MD, unpublished data presented at the International Conference on Toxoplasmosis: Biology, Clinical Practice and Public Health, Copenhagen, Denmark, 23-25 June 2003). In a study of 130 individuals with anti-T-gondii $\operatorname{IgG}$ and IgM antibodies, also from the area of Erechim, who were recruited from June 2003 through June 2004, age > 30 years was among the factors associated with ocular involvement at the time of study enrollment $[\mathrm{OR}=4.9,95 \%$ CI 1.2 20.4, $\mathrm{p}=0.03$ (Dadgostar et al. 2008)].

\section{Disease manifestations}

Among those with ocular toxoplasmosis, age is one of several host factors that are believed to influence severity of ocular toxoplasmosis (Holland 2004) and it is generally believed that ocular toxoplasmosis is a more severe disease at the extremes of age. Although ocular involvement is more common among patients with congenital infections, it is less well established that ocular findings in this population are different than those seen in patients with post-natally acquired infections.

The presence of macular lesions has traditionally been considered to be a sign of congenital toxoplasmosis, but more recent studies show that the presence of macular lesions does not distinguish reliably between congenital and post-natally acquired infections. Macular lesions are present in at least $38 \%$ of patients with serologic evidence of post-natally acquired infection in 
Southern Brazil (C Silveira, unpublished observations). Bosch-Driessen et al. (2002) showed a high prevalence of macular lesions among patients with known congenital infections [11 (46\%) of 24 eyes], but in only a small proportion of their patients was it known whether infection had been congenital or post-natally acquired. This percentage was reported to be statistically higher than the percentage for all other patients in the series, but was not confirmed to be statistically higher than the percentage for those known to have post-natally acquired infections [4 (21\%) of 19 eyes; $p=0.116$, Fisher exact chi square test].

There are some data to suggest that the location of lesions does not occur as a random event. Mets et al. (1996) showed that $52(58 \%)$ of 89 newborns with congenital $T$. gondii infection and ocular disease had macular lesions, which is substantially higher than the number expected if lesions were distributed randomly, considering the fact that the anatomic macula comprises only approximately $5 \%$ of the total retinal area. Other investigators have also shown a disproportionately high frequency of macular lesions among patients with ocular toxoplasmosis (Hogan et al. 1964, Friedman \& Knox 1969, Dodds et al. 2008). Congenital $T$. gondii infection at an early stage of retinal development could favor macular involvement (before vascularization of the peripheral retina is complete). Anatomic and microvascular differences between the macula and the peripheral retina might create a microenvironment that also influences the location of lesions following either congenital or post-natally acquired infections. For example, a study of post-mortem eyes from individuals with no known ocular disease or immune systemic dysfunction showed that macrophages, which participate in host defenses against $T$. gondii infection, were significantly less common in the macula than in the peripheral retina (Yang 2000).

The emphasis on severe ocular disease in patients with congenital toxoplasmosis that can be found in the medical literature may, in part, be a function of ascertainment bias; patients with congenital toxoplasmosis can also have mild ocular disease, but such cases may not come to the attention of physicians. Because of the broad spectrum of ocular findings, and because congenital toxoplasmosis does not seem to be associated with any unique ocular findings, clinical characteristics do not reliably distinguish between individual patients with congenital and post-natally acquired $T$. gondii infections.

Older patients may have not only a higher prevalence of ocular involvement, but ocular disease of greater severity as well. Among 34 reported patients described in two publications that focused specifically on older patients (mean age 67.1 years; median age 67.5 years; range 50-87 years), at least 22 patients had severe disease in terms of multiple active lesions, large lesions [ $>3$ disc areas (DA) in size], prolonged disease ( $>8$ weeks in duration) or a combination of these factors (Johnson et al. 1997, Labalette et al. 2002). In addition, 20 patients (59\%) did not have pre-existing retinochoroidal scars and at least $14 \mathrm{pa}-$ tients (41\%) had anti-T. gondii IgM antibodies, suggesting that infection was recently acquired. Thus, the severity of disease could not be attributed to the cumulative effect of many years of recurrences in this older population.
The two series described above are subject to reporting bias, but various other studies that did not select patients on the basis of age also suggest that older patients have more severe ocular disease. Additional analysis by Holland (2004) of data published by Friedmann and Knox (1969) showed that fewer of their patients aged 2039 years had loss of "useful vision" ( $p=0.099$, Fisher exact chi square test) and a greater proportion of them had brief episodes of disease (shorter than the mean duration of all episodes, $p=0.079$ ) than either younger or older patient groups. Almost twice as many patients older than 40 years of age had prolonged episodes when compared to patients aged $20-39$ years ( $46 \%$ vs. $24 \%$, respectively). In other studies, patients with extensive necrotizing retinopathies, found eventually by PCR or intraocular antibody production to be caused by $T$. gondii infection, tend to be either immunosuppressed or elderly (Fardeau et al. 2002, Moshfeghi et al. 2004).

Severe ocular toxoplasmosis in elderly patients has been attributed to alterations in host immunity (Johnson et al. 1997). Aging is associated with complex changes in both adaptive and innate immune mechanisms that increase the prevalence and severity of many infections in the elderly (Pawelec et al. 1998, Grubeck-Loebenstein \& Wick 2002); changes involve lymphocytes, natural killer cells, macrophages and cytokine production, all of which are known to be involved in host defenses against $T$. gondii.

In an international, multi-center study of 210 patients with active toxoplasmic retinochoroiditis (not selected on the basis of age), there was a relationship between age and lesion size (Dodds et al. 2008). Patients with lesions $>1$ DA in size were older [mean \pm standard deviation (SD), $36.9 \pm 16.8$ years] than those with lesions $\leq 1 \mathrm{DA}$ in size (mean $\pm \mathrm{SD}, 30.7 \pm 13.0$ years, $\mathrm{p}=.006)$. When patients were categorized into age groups $(0-19$ years; 20 39 years; $40-59$ years; $\geq 60$ years), there appeared to be a threshold effect with patients $\geq 60$ years of age having a significantly higher percentage of lesions $>1 \mathrm{DA}$ in size $(77 \%$ vs. $42 \%$ for patients $<60$ years of age; $p=.020$ ). A relationship between older age and the area of "scarring" could be explained by a history of progressive enlargement in older patients having long-standing disease and multiple reactivations, but such factors would not explain a relationship between age and the area of disease activity during a recurrence. It is more likely that the relationship reflects waning immunity among elderly patients, with a decreased ability to limit the proliferation of parasites. There also was a relationship between patient age and signs of inflammation. Eyes with more severe vitreous humor cells and vitreous humor haze were slightly older than those with less severe scores [mean \pm $\mathrm{SD}, 35.6 \pm 15.4$ years vs. $31.0 \pm 14.4$ years, respectively, for cells $(\mathrm{p}=0.056)$ and $36.2 \pm 15.5$ years vs. $31.5 \pm 13.4$ years $(p=0.047)$, respectively, for haze]. When age was considered as a categorical variable, the relationship between age and severity of vitreous humor inflammation remained highly significant for cells; there was an apparent threshold effect, with patients under 20 years of age having less severe vitreous humor inflammation ( $p$ $<.001)$. Holland (2004) had previously hypothesized that 
waning immunity may limit the severity of inflammatory reactions associated with retinal infections in older patients, but these results did not support that hypothesis. Multivariate analysis showed that the observed relationship between older age and more severe inflammation could not be attributed to an indirect effect of larger lesions among older patients.

\section{Recurrences}

A hallmark of ocular toxoplasmosis is the recurrence of active retinochoroiditis from tissue cysts that remain in the retina after initial infection with $T$. gondii. Recurrences usually occur at the borders of retinochoroidal scars, which mark the sites of previous episodes of active disease. The factors that precipitate or facilitate a recurrence are not known, but in a study of 143 patients with toxoplasmic retinochoroiditis in the Netherlands, it was shown that recurrence risk was highest immediately after an episode of active disease, then decreased with increasing disease-free intervals after the episode (Holland et al. 2008). That risk was influenced further by patient age at the first episode of ocular disease and by patient age at the time of the active episode under consideration. Relative risk (RR) of recurrence declined $15 \%$ [RR 0.85 (95\% confidence interval 0.71-1.01), $\mathrm{p}=0.06]$ for each 10 year increase in age at first episode. Patients older than 40 years of age at the time of an active episode were at higher risk of recurrence after an active episode than younger patients [RR 1.74 (1.06-2.86), $\mathrm{p}=0.03]$. Increased risk among older patients may be related to waning of host immunity, as discussed above, with decreased surveillance and control of infection from parasites that emerge from tissue cysts.

In clinical practice, recurrences are seen "less commonly" among older patients with toxoplasmic retinochoroidal scars than among young patients with such scars. A study from Switzerland, for example, found that significantly more recurrences were seen among patients less than 30 years of age than among older individuals (Garweg et al. 2008). In fact, univariate analysis of the Dutch cohort also showed that older age at an active episode was associated with a reduced risk of subsequent recurrence. This apparent contradiction can be explained by the complex interaction between age at first episode and age at any subsequent, active episode. These ages are obviously linked by the interval between first episode and recurrence. In the Dutch cohort, risk of recurrence declined $72 \%$ [RR $0.28(0.22-0.36), p<0.001]$ with each 10 year interval since first episode. Most individuals are infected at a young age and the apparent protective effect of older age actually reflects the strong, protective effect of longer duration since first episode. The increased risk of recurrence associated with older age at an active episode was revealed in the Dutch study when multivariate analysis adjusted for duration since first episode.

The fact that older age at first episode had a weakly protective effect while older age at any given episode (most of which were recurrences in the Dutch cohort) was associated with mores recurrences suggests that risk of recurrence differs after episodes associated with initial infection and subsequent, recurrent episodes, on the basis of factors other than age-related changes in immune function or duration of infection. These factors have yet to be elucidated, but they may still be age-related in some way. For example, it is possible that younger individuals are at increased risk for recurrences following initial infection because of age-specific activities that lead to ingestion of more parasites and thus, a greater parasite burden in the retina, or because of age-specific activities that result in exposure to sources contaminated by more virulent parasite genotypes. Variations in innate immunity might also influence both age of infection and parasite burden after ingestion. The interaction of age-related factors contributes to the variability of recurrence patterns observed by clinicians and interpreted previously as randomness.

\section{Current implications and future research}

Although many of the studies cited in this review show only weak associations, collectively they provide compelling evidence that host age influences risk for ocular involvement, its clinical manifestations and the course of disease. These associations have important implications for prognosis and disease management. For example, long-term, intermittent antimicrobial treatment has been shown to reduce the incidence of recurrences in high-risk populations (Silveira et al. 2002). Elderly patients may be one group that would benefit from that type of secondary prophylaxis. Toxoplasmic retinochoroiditis is usually treated with a finite course of one or more antimicrobial drugs, to hasten the resolution disease activity, and concurrent oral corticosteroids, to reduce the risk of tissue damage by the associated inflammatory response. Large lesions of long duration in elderly patients may reflect prolonged replication of parasites, possibly because of impaired immune function; it may therefore be prudent to withhold concurrent corticosteroids in such cases, to avoid further suppression of host defenses.

There are substantial limitations to past studies, including lack of controls, small sample sizes and the possibility of ascertainment bias; also, past studies are difficult to compare in the absence of standardized measures to describe disease manifestations and outcomes. Additional studies will therefore be important to confirm previous findings; to reconcile the results of some studies that are in conflict with one another; and to expand our understanding of the ways in which host age influences ocular toxoplasmosis. Questions to be addressed include the following: (i) Does ocular toxoplasmosis occur earlier in older individuals with $T$. gondii infection? And does the absolute risk of ocular involvement vary with age? (ii) What is the true prevalence of ocular involvement among children with congenital toxoplasmosis? And what is the true prevalence of ocular disease among individuals with post-natally acquired infection? (iii) Do the clinical manifestations of ocular toxoplasmosis truly differ between age groups? How does age influence the manifestations of ocular toxoplasmosis, in terms of lesion location, lesion size and the associated inflammatory response. (iv) If the severity of ocular toxoplasmosis varies with age, what changes associated with aging are responsible for the observed differences? (v) And what are 
the practical consequences of those differences? Should treatment be adjusted on the basis of patient age? Is there a difference in response to the treatment based on age?

Of particular importance will be controlled studies that compare disease characteristics in unselected populations of elderly patients to younger populations. There will be a need in such future investigations for standard measures to describe disease manifestations and outcomes, which will facilitate comparisons between studies. It may also be important to consider patient age as a confounding variable in any study that compares disease manifestations or response to treatment between groups and adjust analyses accordingly.

In the series of 154 patients reported by BoschDriessen et al. (2002), the time at which infection was acquired remained undetermined in 124 patients, as mentioned above. Knowledge regarding the timing of infection is not necessarily important for the short-term management of individual patients with recurrent toxoplasmic retinochoroiditis and there is as yet no information as to whether the long-term prognosis for involved eyes differs depending on whether patients were infected congenitally or post-natally. Nevertheless, because congenital and post-natally acquired infections are associated with different risks of ocular involvement, continued study of these two populations of patients (as well as studies of the relationship between ocular toxoplasmosis and patient age among individuals with post-natally acquired infections) may provide clues about disease mechanisms that result in toxoplasmic retinochoroiditis, as well as information that is relevant to treatment and disease prevention.

\section{REFERENCES}

Alford CAJ, Stagno S, Reynolds DW 1974. Congenital toxoplasmosis: clinical, laboratory, and therapeutic considerations, with special reference to subclinical disease. Bull N Y Acad Med 50: 160-181.

Bosch-Driessen EH, Rothova A 1999. Recurrent ocular disease in postnatally acquired toxoplasmosis. Am J Ophthalmol 128: 421-425.

Bosch-Driessen LE, Berendschot TT, Ongkosuwito JV, Rothova A 2002. Ocular toxoplasmosis: clinical features and prognosis of 154 patients. Ophthalmology 109: 869-878.

Brezin AP, Thulliez P, Couvreur J, Brézin AP, Thulliez P, Couvreur J, Nobré R, Mcleod R, Mets MB 2003. Ophthalmic outcomes after prenatal and postnatal treatment of congenital toxoplasmosis. $\mathrm{Am}$ J Ophthalmol 135: 779-784.

Burnett AJ, Shortt SG, Isaac-Rentom J, Burnett AJ, Shortt SG, IsaacRenton J, King A, Werker D, Bowie WR 1998. Multiple cases of acquired toxoplasmosis retinitis presenting in an outbreak. Ophthalmology 105: 1032-1037.

Carme B, Tirard-Fleury V 1996. Toxoplasmosis among pregnant women in France: seroprevalence, seroconversion and knowledge levels. Trends 1965-1995. Med Mal Infect 26 (Suppl 3): 431-436.

Couvreur J, Desmonts G 1962. Congenital and maternal toxoplasmosis: a review of 300 congenital cases. Develop Med Child Neurol 4: $519-530$.

Dadgostar H, Silveira C, Jones JL, Lee G, Muccioli C, Belfort R Jr, Holland GN 2008. Risk factors for ocular toxoplasmosis among individuals recently infected by Toxoplasma gondii in Southern Brazil. ARVO Program Book (ARVO Abstract) Program 5529, Abstract A140, Fort Lauderdale, p.356. de Moura L, Bahia-Oliveira LM, Wada MY, Jones JL, Tuboi SH, Carmo EH, Ramalho WM, Camargo NJ, Trevisan R, Graça RM, da Silva AJ, Moura I, Dubey JP, Garrett DO 2006. Waterborne toxoplasmosis, Brazil, from field to gene. Emerg Infect Dis 12: 326-329.

Dodds EM, Holland GN, Stanford MR, Yu F, Siu WO, Shah KH, Ten Dam-van Loon N, Muccioli C, Hovakimyan A, Barisani-Asenbauer T, International Ocular Toxoplasmosis Research Group 2008. Intraocular inflammation associated with ocular toxoplasmosis: relationships at initial examination. Am J Ophthalmol 146: 856-865.

Fardeau C, Romand S, Rao NA, Cassoux N, Bettembourg O, Thulliez P, Lehoang P 2002. Diagnosis of toxoplasmic retinochoroiditis with atypical clinical features. Am J Ophthalmol 134: 196-203.

Friedmann CT, Knox DL 1969. Variations in recurrent active toxoplasmic retinochoroiditis. Arch Ophthalmol 81: 481-493.

Garweg JG, Scherrer JN, Halberstadt M 2008. Recurrence characteristics in European patients with ocular toxoplasmosis. $\mathrm{Br} \mathrm{JOph-}$ thalmol 92: 1253-1256.

Gilbert RE, Dunn DT, Lightman S, Murray PI, Pavesio CE, Gormley PD, Masters J, Parker SP, Stanford MR 1999. Incidence of symptomatic Toxoplasma eye disease: aetiology and public health implications. Epidemiol Infect 123: 283-289.

Gilbert RE, Stanford MR 2000. Is ocular toxoplasmosis caused by prenatal or postnatal infection? Br J Ophthalmol 84: 224-226.

Glasner PD, Silveira C, Kruszon-Moran D, Martins MC, Burnier M Jr, Silveira S, Camargo ME, Nussenblatt RB, Kaslow RA, Belfort R Jr 1992. An unusually high prevalence of ocular toxoplasmosis in Southern Brazil. Am J Ophthalmol 114: 136-144.

Grubeck-Loebenstein B, Wick G 2002. The aging of the immune system. Adv Immunol 80: 243-284.

Guerina NG, Hsu HW, Meissner HC, Maguire JH, Lynfield R, Stechenberg B, Abroms I, Pasternack MS, Hoff R, Eaton RB, Grady GF, New England Regional Toxoplasma Working Group 1994. Neonatal serologic screening and early treatment for congenital Toxoplasma gondii infection. N Engl J Med 330: 1858-1863.

Hogan MJ, Kimura SJ, O’Connor GR 1964. Ocular toxoplasmosis. Arch Ophthalmol 72: 592-600.

Holland GN 1999. Reconsidering the pathogenesis of ocular toxoplasmosis. Am J Ophthalmol 128: 502-505.

Holland GN 2003. Ocular toxoplasmosis: a global reassessment. Part I: epidemiology and course of disease. Am J Ophthalmol 136: 973-988.

Holland GN 2004. Ocular toxoplasmosis: a global reassessment. Part II: disease manifestations and management. Am J Ophthalmol 137: $1-17$

Holland GN, Crespi CM, ten Dam-van Loon N, Charonis AC, Yu F, Bosch-Driessen LH, Rothova A 2008. Analysis of recurrence patterns associated with toxoplasmic retinochoroiditis. Am J Ophthalmol 145: 1007-1013.

Holland GN, Muccioli C, Silveira C, Weisz JM, Belfort R, O'Connor GR 1999. Intraocular inflammatory reactions without focal necrotizing retinochoroiditis in patients with acquired systemic toxoplasmosis. Am J Ophthalmol 128: 413-420.

Jankû J 1923. Pathogensa a pathologica anatomie T. Zv. vrozaneho kolobou zlute skurney oku normal ne velikem a mikrophtalmickem s nalezem parisitu v sitnici. Cas Lek Ses 62: 1021-1027.

Johnson MW, Greven GM, Jaffe GJ, Sudhalkar H, Vine AK 1997. Atypical, severe toxoplasmic retinochoroiditis in elderly patients. Ophthalmology 104: 48-57. 
Jones JL, Kruszon-Moran D, Wilson M, McQuillan G, Navin T, McAuley JB 2001. Toxoplasma gondii infection in the United States: seroprevalence and risk factors. Am J Epidemiol 154: 357-365.

Kimball AC, Kean BH, Fuchs F 1971. Congenital toxoplasmosis: a prospective study of 4,048 obstetric patients. Am J Obstet Gynecol 111: 211-218.

Koppe JG, Loewer-Sieger DH, de Roever-Bonnet H 1986. Results of 20-year follow-up of congenital toxoplasmosis. Lancet 1: 254-256.

Labalette P, Delhaes L, Margaron F, Fortier B, Rouland JF 2002. Ocular toxoplasmosis after the fifth decade. Am J Ophthalmol 133: 506-515.

McAuley J, Boyer KM, Patel D, Mets M, Swisher C, Roizen N, Wolters C, Stein L, Stein M, Schey W, Remington J, Meier P, Johnson D, Heydeman P, Holfels E, Withers S, Mack D, Brown C, Patton D, McLeod R 1994. Early and longitudinal evaluations of treated infants and children and untreated historical patients with congenital toxoplasmosis: The Chicago Collaborative Treatment Trial. Clin Infect Dis 18: 38-72.

Mets MB, Holfels E, Boyer KM, Swisher CN, Roizen N, Stein L, Stein M, Hopkins J, Withers S, Mack D, Luciano R, Patel D, Remington JS, Meier P, McLeod R 1996. Eye manifestations of congenital toxoplasmosis. Am J Ophthalmol 122: 309-324.

Montoya JG, Remington JS 1996. Toxoplasmic chorioretinitis in the setting of acute acquired toxoplasmosis. Clin Infect Dis 23: 277-282.

Moshfeghi DM, Dodds EM, Couto CA, Santos CI, Nicholson DH, Lowder CY, Davis JL 2004. Diagnostic approaches to severe, atypical toxoplasmosis mimicking acute retinal necrosis. $\mathrm{Oph}$ thalmology 111: 716-725.

Ongkosuwito JV, Bosch-Driessen EH, Kijlstra A, Rothova A 1999. Serologic evaluation of patients with primary and recurrent ocular toxoplasmosis for evidence of recent infection. Am J Ophthalmol 128: 407-412.

Papoz L, Simondon F, Saurin W, Sarmini H 1986. A simple model relevant to toxoplasmosis applied to epidemiologic results in France. Am J Epidemiol 123: 154-161.

Pawelec G, Solana R, Remarque E, Mariani E 1998. Impact of aging on innate immunity. J Leukoc Biol 64: 703-712.

Portela RW, Bethony J, Costa MI, Gazzinelli A, Vitor RW, Hermeto FM, Correa-Oliveira R, Gazzinelli RT 2004. A multihousehold study reveals a positive correlation between age, severity of ocular toxoplasmosis, and levels of glycoinositolphospholipid-specific immunoglobulin A. J Infect Dis 190: 175-183.
Rieger H 1951. Zur Klinik der im Erwachsenenalter erworbenen Toxoplasmose. Klin Monatsbl Augenh 119: 459-476.

Ronday MJ, Luyendijk L, Baarsma GS, Bollemeijer JG, Van der Lelij A, Rothova A 1995. Presumed acquired ocular toxoplasmosis. Arch Ophthalmol 113: 1524-1529.

Silveira C, Belfort R Jr, Burnier MJ, Nussenblatt R 1988. Acquired toxoplasmic infection as the cause of toxoplasmic retinochoroiditis in families. Am J Ophthalmol 106: 362-364.

Silveira C, Belfort R Jr, Muccioli C, Abreu MT, Martins MC, Victora C, Nussenblatt RB, Holland GN 2001. A follow-up study of Toxoplasma gondii infection in Southern Brazil. Am J Ophthalmol 131: 351-354.

Silveira C, Belfort R Jr, Muccioli C, Holland GN, Victora CG, Horta BL, Yu F, Nussenblatt RB 2002. The effect of long-term intermittent trimethoprim/sulfamethoxazole treatment on recurrences of toxoplasmic retinochoroiditis. Am J Ophthalmol 134: 41-46.

Sousa OE, Saenz RE, Frenkel JK 1988. Toxoplasmosis in Panama: a 10-year study. Am J Trop Med Hyg 38: 315-322.

Stagno S, Dykes AC, Amos CS, Head RA, Juranek DD, Walls K 1980. An outbreak of toxoplasmosis linked to cats. Pediatrics 65: 706-712.

Stagno S, Reynolds DW, Amos CS, Dahle AJ, McCollister FP, Mohindra I, Ermocilla R, Alford CA 1977. Auditory and visual defects resulting from symptomatic and subclinical congenital cytomegaloviral and toxoplasma infections. Pediatrics 59: 669-678.

Stagno S, Thiermann E 1973. Acquisition of toxoplasma infection by children in a developing country. Bull World Health Organ 49: 627-631.

Wilder HC 1952. Toxoplasma chorioretinitis in adults. Arch Ophthalmol 48: 127-136.

Wilson CB, Remington JS, Stagno S, Reynolds DW 1980. Development of adverse sequelae in children born with subclinical congenital Toxoplasma infection. Pediatrics 66: 767-774.

Wising P 1952. Akut adult toxoplasmos med lymfadenopathi och chorioretinit. Nord Med 47: 563-565.

Wolf A, Cowen D, Paige BH 1939. Human toxoplasmosis: occurence in infants as an encephalomyelitis; verification by transmission to animals. Science 89: 226-227.

Yang P, Das PK, Kijlstra A 2000. Localization and characterization of immunocompetent cells in the human retina. Ocul Immunol Inflamm 8:149-157. 